\title{
PERSPECTIVAS EPISTEMOLÓGICAS ENTRE COMUNICACIÓN Y EDUCACIÓN
}

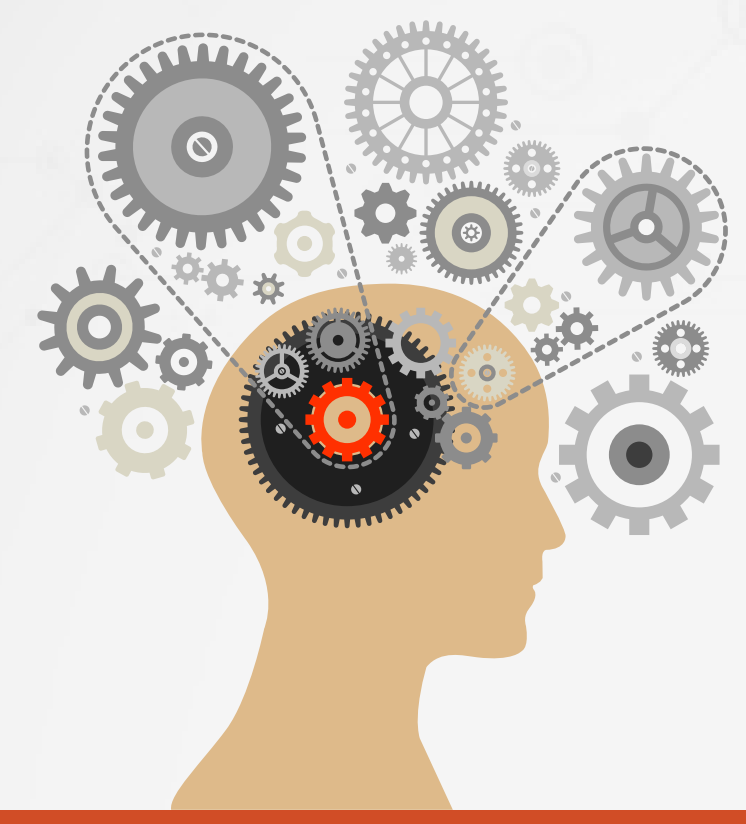

\section{Elssy Yamile Moreno Pérez*}

Recibido. Marzo 22, 2017 - Aceptado. Abril 18,2017

\section{RESUMEN}

Este artículo identifica algunos de los aportes teóricos de autores anglosajones y rusos acerca de la relación entre comunicación y educación. Para lograrlo, se hace inicialmente

una descripción de los conceptos de comunicología, pedagogía y alfabetización mediática. Luego se describen y analizan los vínculos establecidos entre las categorías

mencionadas, así como la manera de asumirlas teórica y empíricamente.

Palabras clave: comunicología, pedagogía, comunicación, educación, alfabetización mediática, pedagogía crítica de la comunicación.

*Docente Investigadora de la Maestría en Comunicación, Desarrollo y Cambio Social de la Universidad Santo Tomás. Comunicadora Social - Periodista de Uniminuto, Magister en Desarrollo Educativo y Social y Candidata a Doctora en Educación de la Universidad Pedagógica Nacional de Colombia. Email: elyamope@yahoo.es 


\section{SUMMARY}

This article identifies the contributions made by some Anglo-Saxon theorists about the relationship between communication and education. To achieve this, initially gives a description of the following concepts: communicology, education and media literacy. Then the links established between the categories mentioned (communication and education) are described and analyzed as well as how to theoretically and empirically assume.

Keywords: Communicology, pedagogy, communication, education, media literacy and critical communication pedagogy.

\section{PERSPECTIVAS EPISTEMOLÓGICAS ENTRE COMUNICAÇÃO E EDUCAÇÃO}

\section{RESUMO}

Este artigo identifica algumas das contribuições teóricas de autores anglo-saxões e autores russos sobre a relação entre comunicação e educação. Alcançar isto, é inicialmente feito com uma descrição dos conceitos de comunicología, pedagogia e alfabetização mediática. Então eles são descritos e eles analisam os laços estabelecidos entre as categorias mencionadas, como também o modo para os assumir teórico e empiricamente.

Palavras chaves: comunicología, pedagogia, comunicação, educação, alfabetização mediática, pedagogia crítica da comunicação. 


\section{INTRODUCCIÓN}

E ste texto se propone indagar sobre la relación comunicación/educación como campo disciplinar de estudio en la literatura anglosajona. Para empezar, a manera de introducción, planteo brevemente un concepto de comunicación, así como las ideas que orientarán el desarrollo del documento.

“La comunicación no sólo es una condición previa para la vida, sino también el criterio de su identificación: un ser vivo es sólo identificable como un ser comunicante (que hereda un código genético, responde a los estímulos ambientales, y así sucesivamente a lo largo de su vida). La vida es igual a la semiosis; es decir, la vida es igual a un proceso en el que las señales están activas o detectables (para ser más precisos: 'signos de vida'). La comunicación así descrita no es en absoluto la exteriorización de un ser vivo preconstituido. Por el contrario, de acuerdo con esta descripción, la comunicación es el ser vivo en si mismo. En el mundo orgánico, comunicación es ser y ser es la comunicación. Comunicar es persistir en el propio ser de uno, para mantener nuestro ser, para confirmarse a uno mismo como ser, conatus essendi” [1] (Petrilli y Ponzio, 2005, p. 519).

Nada mejor para empezar, que esta idea de comunicación expresada por Petrilli y Ponzio. Aquí la comunicación es considerada esencial, parte constitutiva de la naturaleza humana y en modo metafórico, del mundo orgánico en general. Esta es pues una concepción esencialista del fenómeno de la comunicación que bien puede contribuir a la conceptualización posterior de la relación entre educación y comunicación.

La siguiente es una preocupación de orden disciplinar (de la comunicología), que contribuye a la discusión:

"La presuposición de la comunicación como instrumental es el recordatorio sutil de una sentida necesidad de reflejar lo común, de reconocer el sentido cultural de las cosas, en lugar de interrogar tan solo a una racionalidad discursiva. Brant Burleson, estudioso de la comunicación está de acuerdo con esto, cuando dice que nosotros no hemos tomado seriamente la comunicación, no suficientemente en nuestra teoría y en nuestra investigación disciplinaria". (Catt y Eicher-Catt, 2010, p.16)

Tal como lo mencionan Catt \& Eicher-Catt (2010) la comunicación en muchos casos es vista de manera instrumental, asociada más a las técnicas que a la construcción de sentido; pero a la vez, logran que el lector se pregunte por qué estas miradas siguen existiendo, es decir, se interroga por las condiciones culturales que dan lugar a que posturas teóricas y prácticas asociadas a estas se perpetúen. Desde su perspectiva aún es necesario fortalecer los estudios sobre el carácter disciplinar de la comunicación, así como su profundidad y complejidad. Los autores en mención emplean el concepto denominado "communicology" (Catt \& Eicher-Catt, 2010), o comunicología, como se le dirá en adelante; y se refieren a ella como la "nueva ciencia del discurso encarnado", como la ciencia encargada del estudio del fenómeno de la comunicación, planteamiento que se expondrá más adelante.

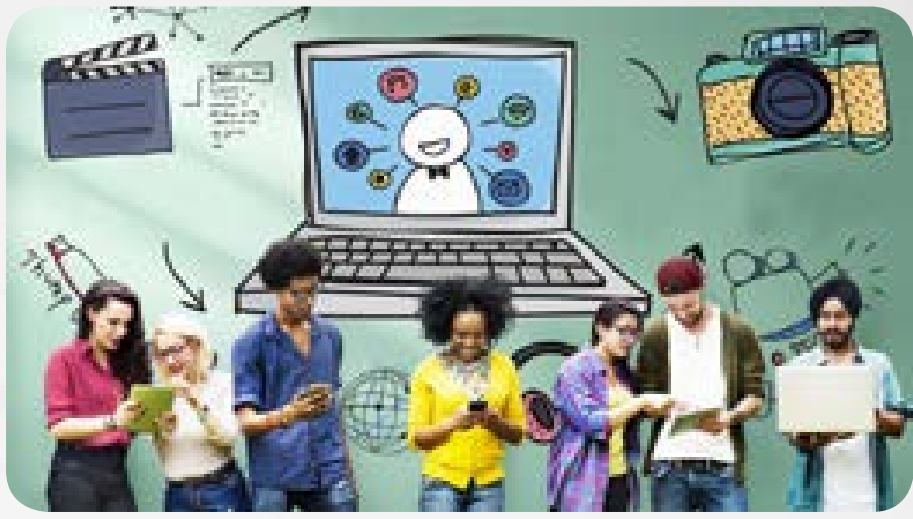

Para acceder al concepto de comunicología, Catt (2003) manifiesta que la preeminencia del modelo informacional de la comunicación (The mathematical theory of communication by Claude E. Shannon and Warren Weaver) y el uso indiscriminado de los conceptos hace que la tarea de construir un cuerpo teórico para la comunicología sea una necesidad imperante; al respecto, ha dicho:

\begin{abstract}
"Al mensaje fue inicialmente asignada la tarea de transportar, transmitir, o contener significado. Inevitablemente, el mensaje sería concebido idéntico al significado. Este desfase de la ciencia sumado a la mera reflexión de sentido común dio lugar a la denominada Teoría de la Información y alcanzó rápidamente asenso sobre la Teoría de la Comunicación en la llamada post-humana, Era de la Información (Radford, 2005). Así, llegamos hoy a la lamentable situación en la que estos términos se utilizan indistintamente: comportamiento, mensaje, significado y comunicación"(Catt, 2003, p.158)
\end{abstract}

En cuanto a la relación entre comunicación y educación hay cierta distancia entre la literatura iberoamericana y la anglosajona. En el primer caso las acepciones se relacionan con las prácticas de comunicación y educación en cualquier escenario social y suele tener un fondo ideológico sobre la emancipación y se soporta en autores como Paolo Freire, lo que no dista substancialmente de la experiencia latinoamericana. En el segundo caso, sus orígenes están centrados en los estudios y usos de la retórica:

"Las raíces históricas de la comunicación educación datan de milenios atrás, sobre el estudio y la enseñanza de estrategias retóricas en las tradiciones clásicas de los griegos y romanos. Como un objeto de estudio formal, la retórica bien podría reclamar el título de la fundación o base de la educación comunicación tal como la conocemos hoy en día. De los escritos de la antigua Grecia y Roma a través de la Edad Media hasta los tiempos modernos, la enseñanza de la retórica ha sido una parte central de la educación occidental."(Sherwyn Morreale, et. Al., 2014, p.345)

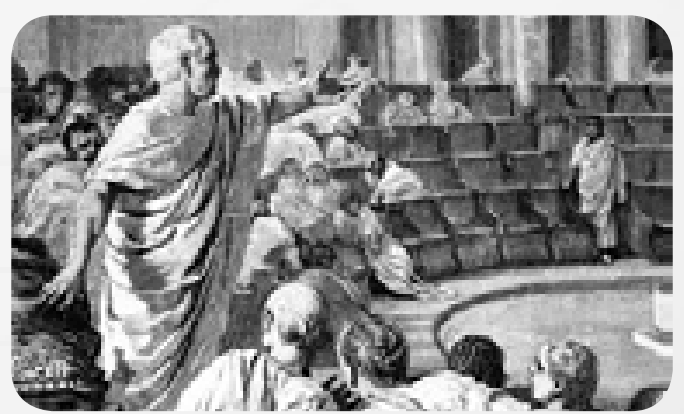



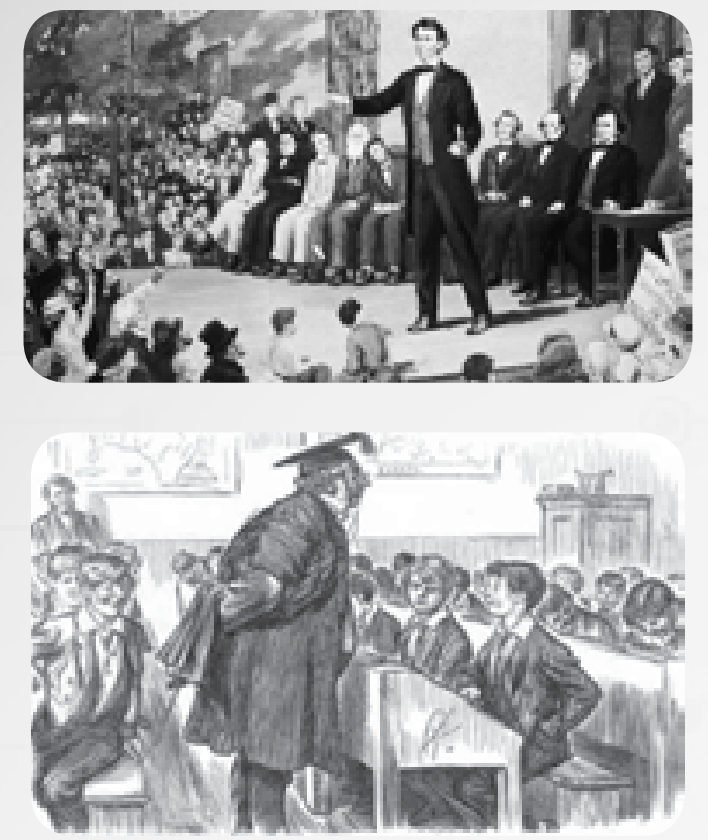

Como es evidente, al menos en principio, la manera de concebir el campo desde esta forma de enunciación no es equiparable con la iberoamericana, en la medida que se refieren a procesos y si se quiere, fenómenos diferentes.

Partiendo de lo expuesto, a continuación se plantea una reflexión a partir de tres líneas ordenadoras de la discusión, para comprender con mayor claridad los posibles elementos de diálogo entre las construcciones teóricas iberoamericanas y anglosajonas, más allá de la mera denominación del campo de estudio.

En este trabajo se pretende identificar y analizar algunos aportes de los teóricos anglosajones a la relación entre la educación y la comunicación, desde una perspectiva epistemológica. El texto se construye a partir de tres líneas de trabajo: Aportes a la definición de comunicología y pedagogía, aportes a la definición de la alfabetización mediática (media literacy), revisión de estudios sobre la educación/ comunicación.

Aportes a la definición de comunicología y pedagogía

\subsection{Comunicología}

Las discusiones sobre la condición disciplinar de la comunicación están presentes en la literatura contemporánea de occidente. Existe una preocupación por definir el soporte epistemológico de esta categoría, así como un interés por delimitar, al menos parcialmente, sus fronteras disciplinarias, sus métodos y sus objetos, por ello, algunos autores, especialmente estadounidenses, rusos, canadienses y mexicanos, han empezado a usar con mayor frecuencia el concepto denominado "comunicología" para referirse a los estudios sobre el fenómeno de la comunicación. En este caso se presentará el trabajo realizado fundamentalmente en Estados Unidos y algún acercamiento a la mirada Rusa.

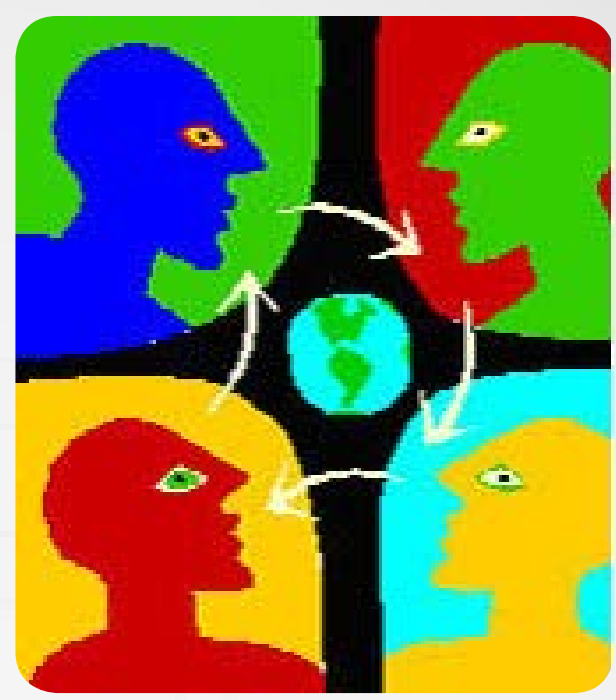

En Russia, Sharkov (2014) en el reciente número de la revista "Communicology", en el artículo introductorio de la publicación, plantea su idea sobre la comunicología, que se cita a continuación:

"En mi definición de autor, la comunicología es un
sistema del conocimiento y actividades constituidas
para obtener nuevos conocimientos sobre la
comunicación, sintetizados en el conocimiento
unido (ciencia): diferentes teorías de comunicación
desarrolladas por diversos autores (por ejemplo, la
teoría de la comunicación de masas, la teoría de
la comunicación intercultural, la sociolingüística,
la teoría de la comunicación igualitaria etc.),
ciencias diferentes con estudios orientados hacia
la comunicación (sociología de la comunicación,
la psicología de la comunicación y otros), teoría y
práctica de la actividad comunicativa en diferentes
aspectos de la sociedad utilizando diferentes
ayudas y temas.

Así, la comunicología incluye la adquisición y la sintesis de nuevos conocimientos acerca de: los tipos y modelos de comunicación, las funciones de los sistemas de comunicación, la estructura y contenido de la comunicación intercultural, y también los métodos de investigación de esta en diferentes aspectos sociales. Además, estudia los problemas étnicos, nacionales, territoriales, las características sociales y privadas de los comunicantes; los componentes lingüísticos, psicológicos, psicolingüísticos, semióticos y semiosociosicológicos propios de la teoría de la teoría de la comunicación intercultural." (Sharkov, F., 2014, p.1)

Según lo expuesto por Sharkov(2014), lacomunicología es una ciencia, un sistema de conocimiento de orden interdisciplinario [2]. Reconoce los elementos propios de la comunicación, los elementos comunicativos que están presentes entre las fronteras con otras disciplinas, así como las relaciones entre unos y otros saberes, es decir, lo que está más allá de cada disciplina, lo que las sobrepasa, lo que surge gracias a su vinculación. En suma, Sharkov concibe a la comunicología como un campo del conocimiento construido con saberes interrelacionados.

En la teoría anglosajona es más común encontrar el término comunicología que en la iberoamericana; de hecho, su aparición se remonta a hace más de ochenta años para referirse a la teoría de la comunicación humana. Lannigan (2008, pág. 855) considera que la comunicología surgió como un concepto en el año 1931 debido a Edward Sapir, quien escribió la entrada "Comunicación" para la Enciclopedia de las Ciencias Sociales. En el caso Iberoamericano, el uso del término se le adjudica al autor hispano-mexicano Eulalio Ferrer (Galindo, 2004). A manera de síntesis Lannigan plantea el concepto de comunicología de la siguiente manera: 
"Comunicología es el estudio crítico del discurso y la práctica, especialmente el cuerpo expresivo que es mediado por la percepción de los signos culturales y los códigos. Utiliza la metodología de la fenomenología semiótica en la que el cuerpo expresivo revela códigos culturales, y los códigos culturales dan forma al cuerpo perceptivo - un continuo, dialéctico, una compleja hélice de giros y vueltas que constituye la reflectividad, la reversibilidad y la reflexividad de la conciencia y la experiencia. Teóricamente y empiricamente, la comunicología se dedica a la descripción, reducción e interpretación de los fenómenos culturales como parte de una comprensión transdisciplinaria. El resultado de la investigación científica es la descripción (en lugar de la predicción) en el que la validez y la fiabilidad son construcciones lógicas basadas en las condiciones necesarias y suficientes de los sistemas descubiertos (códigos), ambos eidéticos (basados en la conciencia) y empíricos (basado en la experiencia). La metodología es intrínsecamente heurística (semiótica) y recursiva (fenomenológica), siendo una lógica en la tradición de Cassirer, Peirce, y Husserl" (Metodología Cualitativa; Métodos de Investigación). "Lannigan (2008, pp 856)

De lo anterior, puede interpretarse que para Lannigan la comunicología hace parte de la tradición en las ciencias humanas que estudian el discurso, así como las manifestaciones semióticas y fenomenológicas de la conciencia y de las prácticas sociales de los sujetos en el entorno en que se desenvuelven.

También, Isaac E. Catt, en la misma línea de Lannigan establece que:

"La comunicología es la nueva ciencia, y la fenomenología semiótica el nuevo paradigma aplicado al mundo social que Bateson predijo y ardientemente buscaba (Donaldson, 1991 [1977]: 157). La comunicología resucita y vigoriza nuestra comprensión fundamental de la comunicación como una posibilidad del discurso. Así hacemos un giro fundamental a la historicidad del mensaje, es decir, la experiencia construida como forma simbólica (...) Considero que lejos de ser una simple cuestión de la producción de mensajes e intercambio de los mismos (La dimensión fática del modelo de Jakobson para el cual el paradigma de transmisión de mensajes sigue comprometido e intelectualmente restringido), la comunicación ofrece el suelo relacional del conocimiento y la información. En la posmodernidad, la comunicación encarnada es un Gestalten de semiosis". (Catt, I. 2003, pp156)

Ambos autores se alejan de la teoría de la información y la transmisión de mensajes y dirigen sus estudios hacia la idea de la comunicología como campo científico centrado en el estudio de la comunicación como un fenómeno plasmado, encarnado en el discurso humano. En ambos casos hay una relación con la fenomenología, puesto que buscan la inteligibilidad teórica y práctica de los fenómenos comunicativos. Esto implica un alejamiento de la comunicación como una condición natural de la especie humana, para acercarse a ella como un objeto de profunda reflexión. Es decir, la preocupación es por el estudio teórico y práctico del discurso, de su esencia, su "eidos".

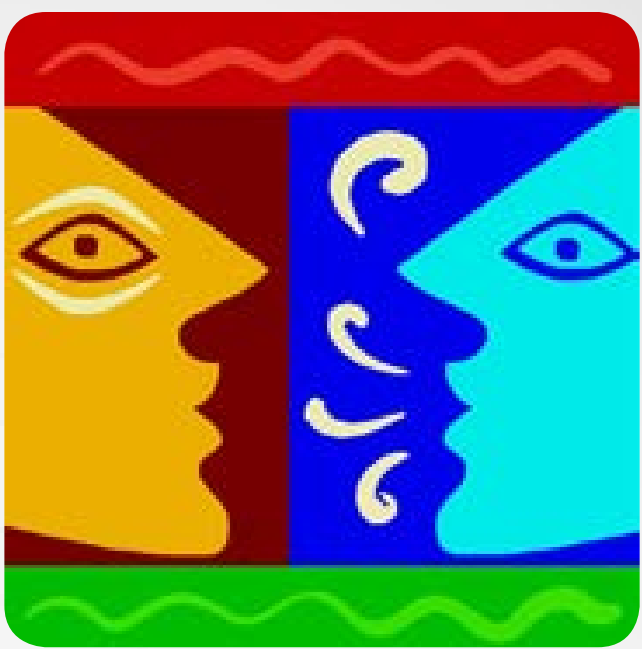

Autores como Lannigan, Catt y Eicher-Catt han luchado contra las ideas que asumen la comunicación desde el sentido común y, como se dijo antes, con las teorías que se soportan casi exclusivamente en el modelo informacional y por consiguiente en la producción y transmisión de mensajes como centro de sus estudios. Ellos (los autores) consideran que la comunicología tiene un carácter trascendental y disciplinar, que suele resultar problemática; sin embargo, afirman:

"La comunicología designa un enfoque holístico de la comunicación que abarca la teoría de la información y los diversos fragmentos del campo. Logra este enfoque, llamar la atención sobre el hecho de que la comunicación es en primer lugar e inevitablemente, una experiencia vivida del cuerpo humano. La palabra comunicología en sí misma puede provocar escepticismo, al igual que la combinación de Augusto Comte del prefijo latino 'socius' con el sufijo griego 'logy' para formar la nueva disciplina de la sociología en 1838. Comte entiende que puede haber una ciencia de la sociedad. Del mismo modo, puede haber una ciencia de la comunicación y el discurso". (Catt y Eicher-Catt, 2010, p.17)

En términos complementarios, cuando Catt \& EicherCatt hablan del discurso, emplean el concepto de "discurso encarnado", que le da unos matices específicos a esta propuesta teórica, puesto que evidencia la complejidad de la misma ya que entiende el discurso como un evento comunicativo cultural, que como tal, se sitúa contextualmente. Estas dos características, así como su uso histórico, permiten recordar que el discurso al que los autores se refieren es también un objeto de interés para diferentes disciplinas tales como la antropología, la filosofía, la lingüística, la sociología, la psicología, la historia, e incluso el psicoanálisis. Argumentan los autores:

"En términos generales como 'la ciencia del discurso encarnado', este enfoque crítico-interpretativo interroga al carácter reversible, recíproco y reflexivo del 'cuerpo expresivo y perceptivo', entendido como el punto esencial de la mediación entre nosotros y los signos culturales y códigos del discurso bajo la cual ineludiblemente vivimos. Comunicología es, por lo tanto, una teoría coherente y una metodología que explora el terreno existencial de que la subjetividad e intersubjetividad emergen como un proceso semiótico encarnada. Por lo tanto, el ejemplo de paradigma de la comunicología es la fenomenología semiótica, una lógica sintética del discurso que combina la sabiduría y metodologías de dos grandes tradiciones de las ciencias humanas.

La semiótica es el estudio de los signos y los códigos que configuran la cultura y que organizan la existencia social. La fenomenología estudia la lógica de las 'appeareances' y cómo se presentan en la conciencia. Todas las convenciones perceptuales [appeareances] tienen forma, en otras palabras, estas formas se entienden mejor 
como signos. Todos los signos están integrados en sistemas de signos o códigos que adicionalmente se combinan dentro de relatos y mitos que crean las órdenes de significación de nuestras experiencias culturales. En resumen, la fenomenología semiótica investiga el devenir del signo dentro de las prácticas discursivas, el nacimiento originario de la conciencia en el sentido descrito por el fenomenólogo Maurice Merleau-Ponty. Los signos siempre tienen una historia en la conciencia, y, mediante la deconstrucción de esta experiencia, es que la formación de conceptos y relaciones pueden ser revelados. Es en la realización o encarnación [embodiment] de los signos que radica la coherencia de la investigación en comunicología”. (Catt y Eicher-Catt, 2010, p.17)

Según estos planteamientos el discurso no es externo al ser humano, es concebido como constitutivo del ser y representado mediante códigos y signos que en su elaboración y articulación generan permanentemente nuevos órdenes de significación. Es decir, no hay comunicación sin discurso y el discurso es mucho más que el texto escrito o verbalizado, es la construcción colectiva de sentido que se hace sobre la vida misma, sobre los asuntos humanos. En el desarrollo de este planteamiento, Catt y Eicher-Catt (2010) y con base en autores como Ruesch y Bateson identifican cuatro niveles, escenarios de lo humano, desde los que puede ser estudiado el fenómeno de la comunicación:

"Estos niveles también se describen en otras partes de la literatura sociológica y semiótica, como en la obra de Norbert Wiley en la 'auto-semiótica', que a su vez, es derivado de un cuidadoso estudio de la filosofía de la comunicación en el pragmatismo americano (...) los niveles son: comunicología intrapersonal (auto dominio), la comunicología interpersonal (dominio sí mismo - otros), la comunicología social (dominio de grupo, de organización), y la comunicología cultural (dominio intergrupo), respectivamente. Los niveles forman una jerarquía dependiente en el que un nivel se distingue del siguiente basado en las restricciones semióticos y la creciente complejidad." (Catt y Eicher-Catt, 2010, p. 19)

Los niveles enunciados resultan pertinentes a la hora de comprender el fenómeno de la comunicación, en la medida que pasan por el reconocimiento de los escenarios en los que esta transita y no en objetos fragmentados de una y otra disciplina.

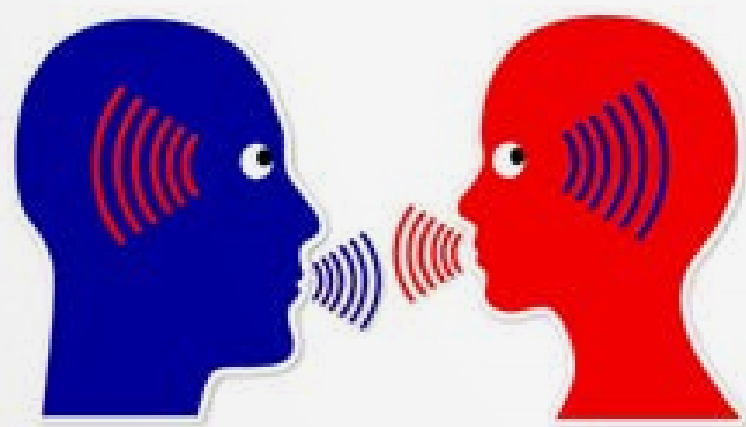

Los estudios sobre el fenómeno de la comunicación gozan de un interés inusitado en la actualidad. Si bien desde una mirada positivista la dificultad para encontrar un objeto y un método único para una disciplina resulta ciertamente problemático, según lo expuesto hasta ahora, en el caso de la Comunicología, este no sería un problema sino por el contrario una ventaja que parte del supuesto de la imposibilidad de entender un fenómeno social de manera aislada, sin reconocer los múltiples matices que este encarna, en tanto humano. Así, según Catt \& Eicher-Catt (2010) esta "nueva ciencia", joven aún, es ciertamente reconocida como tal en el marco de las ciencias sociales y humanas, cada vez se suman a su estudio más comunicólogos en diferentes lugares del mundo. Dicen los autores:
"Ya formalmente aceptado en la escena internacional como la disciplina cultural más reciente o la ciencia humana, la comunicología, sin embargo, sigue siendo un paradigma fresco que invita a nuevos participantes (...) Aún hay espacio para el optimismo, ya que hay comunicólogos que se dedican a la idea de que la calidad de vida se puede mejorar mediante una mejor comprensión del proceso semiótico cultural y del evento fenomenológico encarnado de la comunicación. Por primera vez en la historia, tal entendimiento procede hacia el interior desde las manifestaciones de la conciencia en la cultura humana y hacia fuera de nuestro interés único en la experiencia humana de la comunicación. Por lo tanto, no abogamos por una sola teoría integrada de la comunicación. Estamos a favor de la disciplina coherente.

La comunicología toma en serio las limitaciones y posibilidades del discurso. Por lo tanto, no estudia el mundo de la vida de la comunicación parcial, limitado por sus numerosos sub-disciplinas dispares, restringida ulteriormente por su idealización de la tecnología y su fascinación con los métodos, lo que refleja los déficits de atención multitarea de la posmodernidad, y finalmente consumida en la simplicidad de sus fragmentos de mensajes. En cambio, esta nueva disciplina surge en pleno reconocimiento de la profunda carga de la ciencia humana: Para recuperar de forma continua y resucitar al humano en nuestros objetos"(Catt y Eicher-Catt, 2010, p.25)

En los estudios de Lannigan (1988, 1992, 1995, 2010, entre otros) y los de Catt \& Eicher-Catt (2003 y 2010) aparece también un elemento conceptual fundamental en esta discusión y es "el código". Incluso, la comunicología misma, como teoría, sería también un código, puesto que debe contener unas reglas específicas y unos elementos constituyentes de como los códigos son semánticamente interpretables. Sin embargo, en función de la comunicología, Isaac Catt considera que:

"Un código se define como un sistema de signos junto con las reglas para su uso. El mensaje como signo pertenece a un sistema, un código que especifica información como conocimiento. Con este reconocimiento, se ha pasado de la teoría como explicación a la teoría como la comprensión. Sin embargo, la función meta-semiótica aquí solo está asegurada por el uso fático convencional en el mundo social intersubjetivo. Por lo tanto un código se ve limitado por el contacto y, si esto es así, un mensaje debe ser no solo una medida de tiempo diacrónico, sino también el espacio sistemático. El mensaje solo se realiza en contextos sociales". (Catt, 2003, p. 162)

Desde la perspectiva de Lannigan, comprender la importancia del código en los estudios sobre comunicología es fundamental, puesto que: "Cuándo y dónde los signos y códigos de la cultura impacten sobre la percepción de los modos expresivos corporales, tenemos un fenómeno comunicológico susceptible a ser investigado, interpretado, deconstruido, refigurado, y descrito."(Lannigan, 2007, p. 213). De manera complementaria, Catt afirma:

"Los científicos humanistas estudian la codificación de los mensajes en la sociedad, así como las teorías anticipadas que son los mismos códigos. Pero, en ningún caso debemos presumir que nuestros paradigmas científicos son un reflejo de la realidad vivida, al igual que el modelo de transmisión de mensajes. La ciencia es solo una manera de hacer la realidad inteligible, es decir comunicable (Urban, 1939: 228-267). La comunicabilidad es esencial para la humanidad, pero es de suma importancia recordar que la comunicación es siempre in-formada por un contexto de relaciones código a código. El contexto nos proporciona un esquema semiótico construido de la realidad social. La realidad social está llena de ambigüedad. Entre otras cosas, esto significa que no podemos saber con certeza que hemos logrado con la comunicación, ya que es 
en última instancia una posibilidad abductiva, una 'mejor estimación', no una certeza deductiva ni siquiera una probabilidad inductiva”. (Catt, 2003, p. 153)

Como vemos, Catt (2003) nos recuerda también que no puede perderse de vista el sentido de la teoría, no como lupa para leer la realidad, ni para replicarla al pie de la letra, sino como herramienta para comprenderla, para asirla.

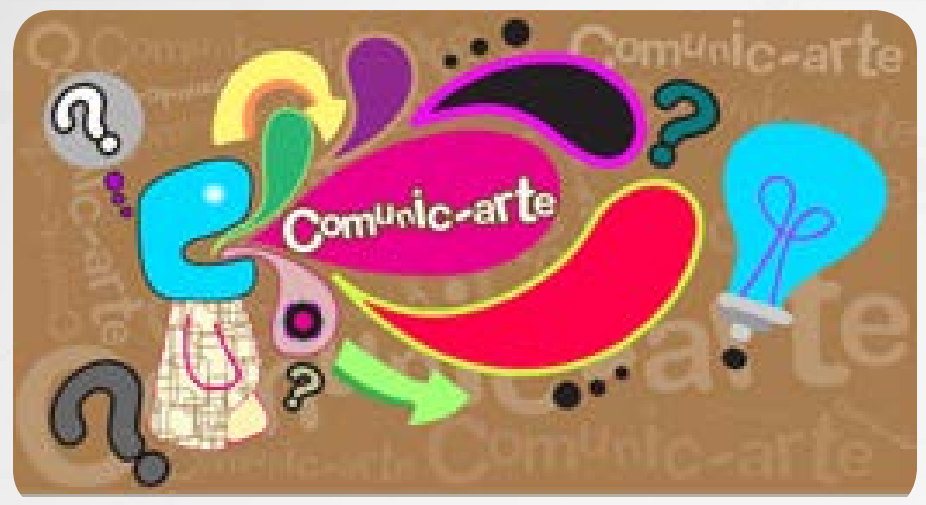

\subsection{Pedagogía}

Este apartado se desarrolla, bajo los aportes de Hamilton, quien hace una revisión del uso del concepto de pedagogía en la actualidad, las acepciones más comunes que suelen encontrarse en diccionarios como en de Oxford, ampliamente aceptado por la comunidad docta, y en el uso académico diario. En cuanto a las raíces del concepto, Hamilton afirma que:

"El tallo de la raíz de la pedagogía está constituido por un par de palabras griegas clásicas que abrazan la infancia y el liderazgo, y ambas están vinculados por la adición del sufijo 'gy' que equivale a 'lógica'. En términos más simples, la pedagogía denota 'la lógica de llevar, liderar niños' (...) Igualmente, la pedagogía podría referirse a la instrucción, la disciplina, el entrenamiento; un sistema de formación introductoria; un medio de orientación'. Estos usos, sin embargo, son descritos por el DEO como, respectivamente, "raros» $y$ «obsoletos» (...) el OED ofrece una definición actual: 'el arte, la ocupación, o la práctica de la enseñanza. También: la teoría o principios de la educación; un método de enseñanza basado en una teoría 'tal'. Además, la pedagogía fue practicada por los pedagogos que tuvieron la tarea de trabajar de acuerdo con la lógica de los niños que llevan”. (Hamilton, 2009, p.6)

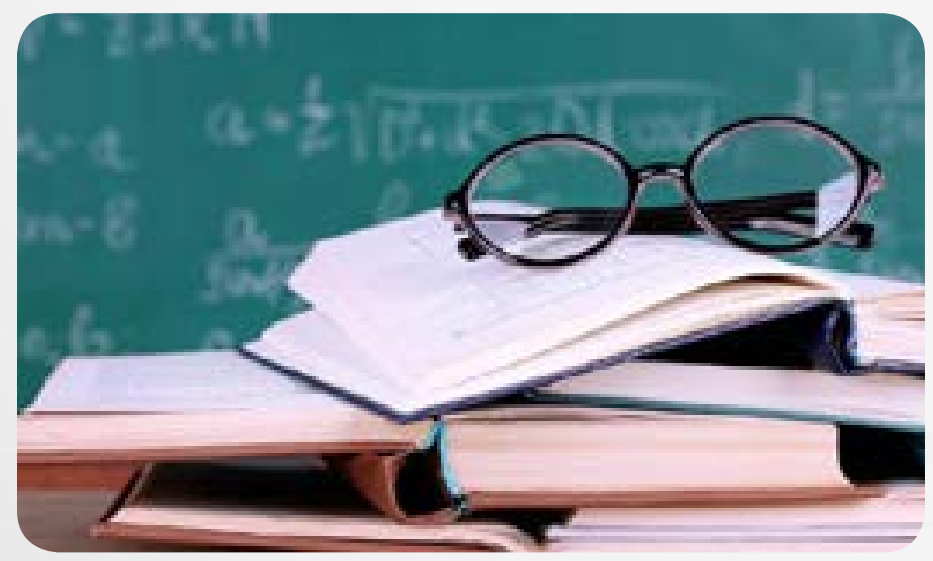

Las definiciones citadas por Hamilton evidencian dos cosas, la primera, que los usos del concepto pueden ser realmente disímiles y la segunda, que no hay claridad frente a su naturaleza, es decir: si es una teoría o una metodología y adicionalmente cuando se refiere a "dirigir los niños", ¿Hacia dónde se supone que se dirigen? ¿Hacia un lugar mejor o hacia un lugar establecido?, es decir, ¿Es la pedagogía confundida aquí con la escolarización como sistema para que los sujetos, especialmente los niños, ingresen al mundo de la cultura, al sistema cultural, a sus reglas, a sus códigos, a su gramática?

\begin{abstract}
"La palabra pedagogía se utiliza cada vez más en los textos en inglés. Su aplicación, sin embargo, no siempre es clara, ya que los autores se basan en diferentes usos heredados. Actualmente, sirve como un sinónimo de la educación; o puede reflejar otras definiciones que han surgido a partir de su integración, a través del tiempo, en el mundo de la educación pública”. (Hamilton, 2009, p.5)
\end{abstract}

Aunque en esta introducción nos referimos a cierto desarrollo histórico del concepto de pedagogía, el propósito no es describir cada una de las escuelas, modelos y sus respectivas construcciones conceptuales sobre esta, sino los usos modernos del concepto. Se parte diciendo que en la literatura anglosajona aparece el significado de educación en contraste con el de pedagogía. Es decir, no suele relacionarse directamente educación y pedagogía en un mismo discurso.

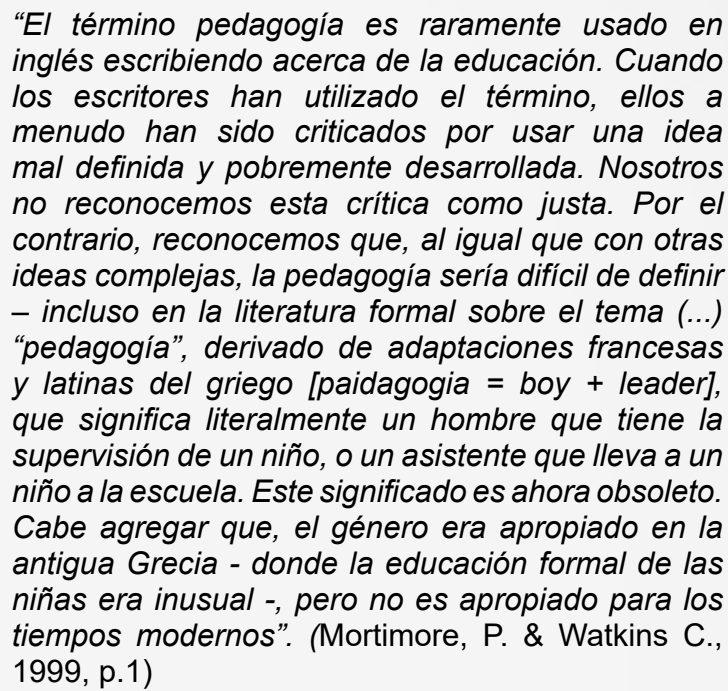

De hecho, hablar de pedagogía en la teoría anglosajona implica pensar también en los problemas de lenguaje y traducción, puesto que el uso de los conceptos es ciertamente disímil entre diferentes culturas. "En los días modernos, el término 'pedagogía' es más común en algunos países europeos, en particular, en Francia, Alemania y Rusia - hablando de comunidades académicas [que hablan inglés]", (Mortimore, P. \& Watkins C., 1999, p.1). A manera de ejemplo, en el caso francés se refieren a la educación, pero usan diferentes acepciones del término según sea el caso y la conceptualización está relacionada con la investigación sobre ciudadanía y por supuesto, en específico, con la conceptualización sobre educación pública francesa.

A manera de ejemplo sobre las dificultades de traducción, puede presentar el caso francés y el caso ruso:

"[En francés] éduquer significa aparecer como bueno, como formalmente educado y bien éduqué significa una buena educación o buenas maneras ('educar' en inglés tiene dos sentidos también, pero este último predomina). La raíz de la palabra rusa para la educación, 'obrazovanie', significa 'forma' o 'imagen' y no, como en la versión latina, un 'liderazgo'. [En ruso también] 'obrazovanie' es inseparable de 'vospitanie', una idea que no tiene equivalente en inglés ya que combina el desarrollo personal, la moral pública y privada, y el compromiso cívico; mientras que en Inglaterra estos tienden a ser tratados como dominios separados e incluso contradictorios. Así mismo, 'obuchenie', que se traduce generalmente como 'instrucción dirigida por el maestro' señala tanto el aprendizaje como la enseñanza”. (Alexander R., 2009, p. 925) 
Cuando Thiessen (et al.) escribió el documento "Perspectives on Pedagogy" aparecían cerca de 77.500 entradas en Google Scholar, desde 2008 hasta septiembre de 2012 (Thiessen et al., 2013, p.2). Unos años después, en la misma herramienta, aparecen más de 580.000 resultados, incluyendo citas, en español y más de 1.040 .000 en inglés y si se hace una revisión diaria el uso aumenta constantemente. Lo que implica que su comprensión es cada vez más compleja, por la variedad de fuentes y por la diversidad de formas de comprenderla. El creciente uso del término "pedagogía" y la proliferación de acepciones sobre el mismo es innegable; al respecto, los autores afirman que:

"Con tantas pedagogías que circulan en la literatura, no siempre es evidente la forma en que individual o colectivamente encarnan un significado particular. Para complicar aún más esta búsqueda, la comprensión de la idea de pedagogía es más difícil debido a la variabilidad en su forma de aplicación y a su aparente capacidad de intercambio con nociones tales como la enseñanza o instrucción. Sin embargo, algunos estudiosos han ofrecido información valiosa sobre el significado de la pedagogía que nos ayudan a dar sentido a la reciente proliferación de este concepto en la literatura". (Thiessen et al., 2013, p.3)

Adicionalmente está la confusión suscitada debido al uso indiscriminado de los conceptos de pedagogía y educación, para referirse a los mismos fenómenos. Reduccionismo que es común a la teorización iberoamericana sobre la materia. Dice Hamilton:

“(...) Como Freire reconoció, la educación es ampliamente considerada como una institución [la escuela] y no como un proceso. $Y$ este problema se agrava en inglés, siempre que se utilice la palabra educación como sinónimo de escolaridad, por ejemplo, cuando los autores escriben sobre un sistema educativo nacional, cuando, de hecho, deberian referirse al sistema escolar. El riesgo es que los padres creen que sus hijos han entrado en el sistema educativo en el que, como consecuencia paradójica, simplemente no reciben una educación. Esto no tiene por qué surgir, por ejemplo, en entornos de habla alemana porque Pedagogik [educación] puede distinguirse de Schulpedagogik [instrucción]". (Hamilton, 2009, p.6)

Hamilton hace un recorrido por el uso del concepto de pedagogía en Inglaterra y Alemania, sin embargo, manifiesta su preferencia por el concepto de pedagogía, asociado a la complejidad de lo público:

"En pocas palabras, los significados se superponen, lo que complica cualquier análisis. Sin embargo, conservo una preferencia para considerar que la pedagogía tiene valor al denotar las complejidades superpuestas de lo público (...) Y lo hago por una razón permanente - un discurso histórico que examina las nociones de liderazgo, instrucción, orientación, gestión, formación moral, la cultura, etc., es en última instancia, más completo que un análisis estrictamente restringido que se refiere a la enseñanza ya sea como 'mal necesario' todavía prescindible (citado en Bruner 2006, vol. 2, 183), un contagio misterioso que nubla el nuevo amanecer de la llamada sociedad del aprendizaje o del conocimiento". (Hamilton, 2009, p.15)

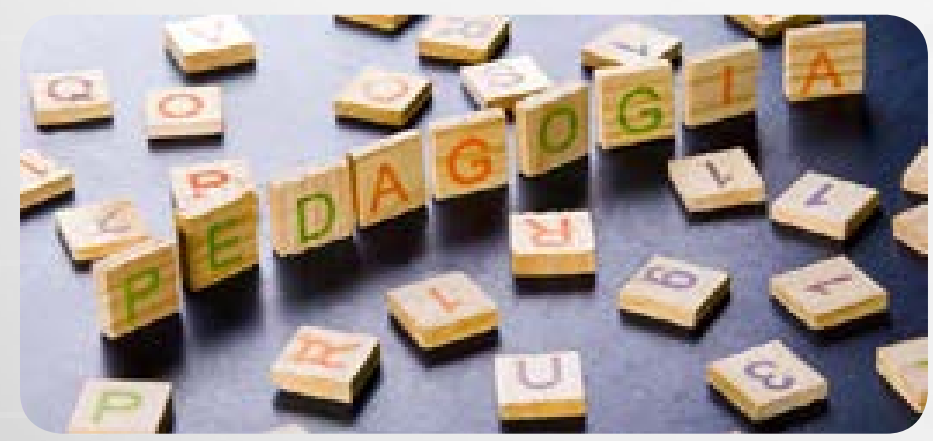

Según Loughran, otro reduccionismo del que es provista la pedagogía es el de equipararla al concepto de enseñanza; afirma:

"Pedagogía es una construcción importante en la educación que se ha definido, interpretado y utilizado de muchas maneras en la literatura educativa. En gran parte de la literatura, la pedagogía es retratada como un sinónimo de enseñanza; lo que puede considerarse como estrecho y superficial. (...) La pedagogía necesita ser conceptualizada como rica, compleja y sofisticada, y al hacerlo, pone de relieve por qué debe ser vista como mucho más que un sinónimo de enseñanza”. (Loughran, 2013, p. 118)

Autores como Vavrus, y Bartlett plantean un concepto de pedagogía también muy cercano al de enseñanza, pero afirman que es marcadamente contextual, es decir que adquiere sentido y valor según el momento histórico y el grupo social que hace uso de este. Lo que podría decirse casi sobre cualquier concepto usado para leer - entender la sociedad. Afirman:

"La pedagogía está profundamente influenciada por las condiciones culturales y materiales en las que enseñan los maestros y las diferentes perspectivas sobre la producción y difusión de conocimientos en poder de los educadores en diferentes ubicaciones sociales”. (Vavrus, F. \& Bartlett L., 2012, p.636)

Otros autores plantean una idea de pedagogía más holística, referida al conjunto de saberes sobre la educación, no sólo sobre la enseñanza: "[La pedagogía] abarca tanto el acto de enseñar, como sus teorías contingentes y los debates acerca de, por ejemplo, el carácter de la cultura y la sociedad, los fines de la educación, la naturaleza de la infancia y el aprendizaje y la estructura del conocimiento" (Robin, A. 2001, pág. 513)

\subsection{Aportes a la definición de la alfabetización mediática (media literacy)}

Este apartado es importante por dos razones; la primera, porque muchas de las experiencias actuales que se enmarcan en la comunicación educativa emplean los nuevos medios para desarrollarlas y la segunda, porque en la relación que se establece entre comunicación y educación, la alfabetización de los públicos ha sido una constante. El acaecimiento de los medios de comunicación y de los denominados nuevos medios ha generado intereses de diversos órdenes en el mundo tales como políticos, económicos, sociales, etc. Mientras que en el mundo aún existen sujetos que no han tenido acceso a procesos de alfabetización clásica (lectura y escritura), cientos de actores del mundo académico se preocupan por la denominada alfabetización mediática o media literacy como se le denomina en el mundo anglosajón. Al respecto comenta, Lin (2013):

"Con la llegada de las nuevas tecnologías de la comunicación, el papel de los medios de comunicación en una sociedad ha estado cambiando, lo que ha llevado a los investigadores a reconstruir el significado de la alfabetización clásica a la nueva alfabetización mediática. Ha habido esfuerzos continuos, de parte de investigadores, educadores y políticos, para entender los nuevos medios de comunicación y promover la importancia de convertirse en alfabetizado en nuevos medios. La comprensión fundamental de lo que es la nueva alfabetización mediática sigue siendo poco clara". (Lin, T.-B., et. Al. 2013, p.160) 


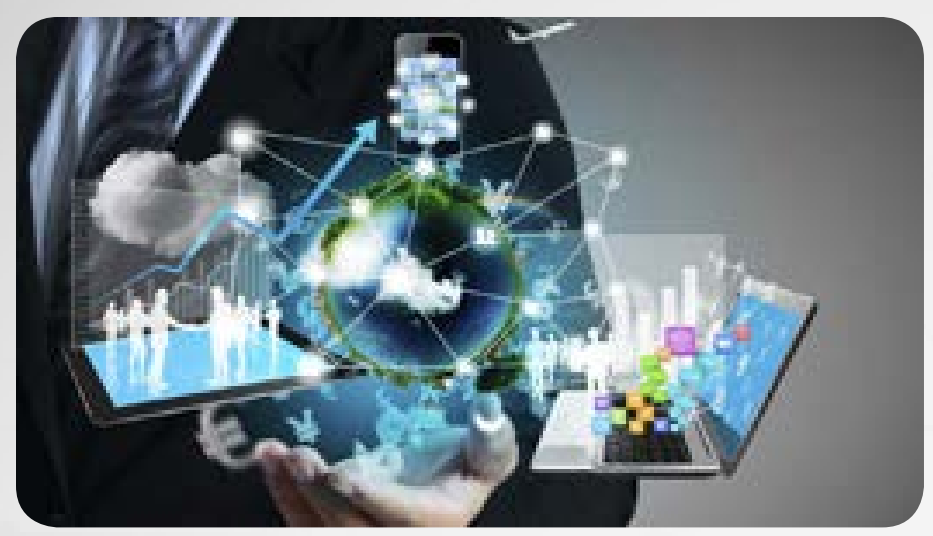

La hipótesis de Lin (2013) es que la alfabetización mediática no es un fenómeno reciente, es decir, que no corresponde sólo a los "nuevos medios" sino que existe casi desde que el primer medio de comunicación de masas existió y que se re-ajusta cada que un "nuevo medio" gana importancia en la agenda mundial:

"La noción de alfabetización mediática ha existido en la cultura occidental durante mucho tiempo (...) El principio de la alfabetización mediática se remonta a la primera mitad del siglo 20, cuando Leavis y Thompson (1933) propusieron enseñar a los estudiantes cómo distinguir la alta cultura y la cultura popular a través de la educación en el Reino Unido. En este enfoque proteccionista estos defensores tenían por objeto promover la alta cultura para luchar contra el creciente aumento de la cultura popular en la era de los medios (Buckingham, 2003).

En 1950, la alfabetización mediática fue introducida a los Estados Unidos con el reconocimiento del creciente impacto de los medios de comunicación, como la radio y la televisión en la vida diaria y la escolaridad de las personas (Schwarz, 2005).

Una tendencia general del desarrollo de la alfabetización mediática, así como la nueva alfabetización mediática es que la importancia de esta es llevada otra vez a la agenda educativa cuando hay una nueva tecnología de los medios que causa ansiedad colectiva en la sociedad (Lin, 2010). La aparición de nuevos medios de comunicación también planteó preocupaciones entre el público en varios países. Por ejemplo, hay un interés recientemente y en crecimiento sobre el tema de la alfabetización mediática en varios países del este de Asia, como China (incluyendo Hong Kong), Taiwán, Japón y Corea desde finales de 1990 (Cheung, 2009)" (Lin, T.-B., et. Al. 2013, p.161)

En cuanto al surgimiento y uso del término "alfabetización mediática" (Media Literacy) Buckingham (2003), aporta más datos:

\begin{abstract}
"Los defensores de la educación para los medios han invocado frecuentemente la noción de 'alfabetización' en un intento de definir y justificar su trabajo. El uso del término en este contexto se remonta al menos a la década de 1970, cuando se introdujeron una serie de planes de estudio en su mayoría de corta duración tales como 'alfabetización televisiva' en los Estados Unidos (Anderson, 1980). En América del Norte en general, el término es aún usado con frecuencia para referirse a la 'educación para los medios'. La referencia a la alfabetización llegó a la orden del día en el Reino Unido a finales de la década de 1980, en parte como resultado de los intentos de integrar la educación en medios dentro de la enseñanza de Inglés" (Buckingham, D. 2003, pág.35)
\end{abstract}

Sin embargo, Buckingham (2003) plantea una postura crítica al respecto, en tanto considera que es bastante impreciso usar el término "alfabetización mediática" teniendo en cuenta los usos clásicos del término "alfabetización" y la imposibilidad de relacionar la gramática del medio con la gramática del lenguaje:
“El término 'alfabetización mediática' se refiere a los conocimientos, habilidades y competencias que se requieren con el fin de utilizar e interpretar los medios de comunicación. Sin embargo, la definición de la alfabetización mediática está lejos de ser sencilla. Hablar de 'alfabetización' en este contexto parecería implicar que los medios pueden, en cierto sentido ver y emplear formas de lenguaje - y que podemos estudiar y enseñar 'lenguas' visuales y audiovisuales de una manera similar a la lengua escrita (...) la analogía con el lenguaje escrito - y el término 'alfabetización mediática' - es simplemente demasiado imprecisa, si no positivamente engañosa. Algunos estudiosos de la alfabetización, por ejemplo, precavidos contra este uso más bien suelto y metafórico del término, argumentan que difumina las distinciones necesarias entre la lengua escrita y otros medios de comunicación"(Buckingham, 2003, p. 36).

Según Lin (2013), con la llegada de los medios emergentes en los comienzos del siglo XXI, la alfabetización tradicional ya no es suficiente para que el individuo sobreviva en el entorno actual:

"Wu y Chen (2007) argumentaron que los medios de comunicación no se limitan a la formación de nuestra cultura; son nuestra cultura. En otras palabras, los nuevos medios de comunicación desempeñan un papel indispensable en las sociedades humanas y los individuos necesitan dotarse de nuevas competencias para poder participar plenamente en el nuevo entorno de los medios de comunicación (Cope y Kalantzis, 2000)." (Lin, T.-B., et. Al. 2013 p.161)

Sobre la cita anterior vale la pena detenerse en la afirmación de Wu y Chen (2007), puesto que aparecen dos elementos problemáticos, el primero es que se asume a la cultura como producto del medio (y no al contrario) y se afirma que el medio es la cultura misma. Las dos afirmaciones, le otorgan al medio una condición que supera lo técnico y lo vuelve parte constitutiva de la vida en sociedad. Desde mi perspectiva hay cierta idealización del medio y de su rol en la sociedad.

Por otro lado, existen también definiciones construidas por organizaciones preocupadas por el uso de los medios en la educación como es el caso de la Asociación de Ontario para la Alfabetización Mediática y OFCOM:

"En 1989, la Asociación de Ontario para la Alfabetización Mediática (AML) ofreció esta definición: La alfabetización mediática se ocupa de desarrollar una comprensión informada y crítica de la naturaleza de los medios de comunicación, las técnicas utilizadas por ellos, y el impacto de estas técnicas. Es la educación que tiene como objetivo aumentar la comprensión y el disfrute de los estudiantes frente a cómo funcionan los medios, cómo producen significado, cómo están organizados y cómo construyen la realidad. La alfabetización mediática también tiene como objetivo proporcionar a los estudiantes la capacidad de crear productos de los medios." (Duncan, B. 2005, p 31)

OFCOM, el regulador independiente de la industria de las comunicaciones en el Reino Unido plantea también una definición de alfabetización mediática (media literacy), que es entendida como "la habilidad para acceder, entender y crear comunicaciones en una variedad de contextos". Al respecto, las investigadoras Sonia Livingstone, Elizabeth Van Couvering y Nancy Thumim, consideran que: 
"Con la creciente importancia de los medios de comunicación, la información y las comunicaciones en la sociedad, la alfabetización mediática puede cumplir con tres objetivos clave, i) contribuyendo a la democracia, la participación y la ciudadanía activa; (ii) a la economía del conocimiento, la competitividad y la elección; y (iii) al aprendizaje permanente, la expresión cultural y la realización personal".(Livingstone, S., et al. 2004, p.7)

Sobre alfabetización mediática existe más información que sobre alfabetización cultural. Probablemente por la preocupación actual frente a la relación hombre - escuela - tecnologías de la información y la comunicación. Autores como Potter (2014), han realizado una revisión del estado de la cuestión, en la que encuentran que:

"El tema de la alfabetización mediática ha generado una variedad bastante grande de la escritura. Algunos estudiosos se han centrado en el nivel cultural y han argumentado que los medios crean una falsa conciencia en la sociedad para promover su propia agenda ideológica (Alvarado y Boyd-Barrett, 1992; Bazalgette Bevort, y Savino, 1992; Brow 1991: Masterman, 1980, 1985) y la alfabetización mediática es, por tanto, vista como una manera de empoderar a las personas para poder leer mejor los textos mediáticos (Buckingham, 199; Messaris, 1994; Meyrowitz, 1998; Potter, 2001; Zett, 1998) o ser capaz de identificar las ideologías subyacentes a los mensajes de los medios (Buckingham, 1998: Lewi y Jhally, 1998). Los eruditos han escrito sobre la necesidad de que las instituciones educativas presten más atención a la alfabetización mediática (Anderson, 1980; Bazalgett 1989; Blanchard y Cristo, 1993; Buckingham, 1998; Cristo y Blanchard 1994; Hobbs, 1997; Kubey, 1997; Limburg, 1994; McLaren, Martillo, Sholle y Reilly, 1995; Piette y Giroux, 1997; Sholle y Denski, 1994; Silverblat 1995; Sinatra, 1986). Y algunas organizaciones han abogado por una política pública más fuerte sobre la alfabetización mediática (Aufderheide, 1993: Asociación de Comunicación Oral, 1996)".

(Potter, WJ 2004, p.267)

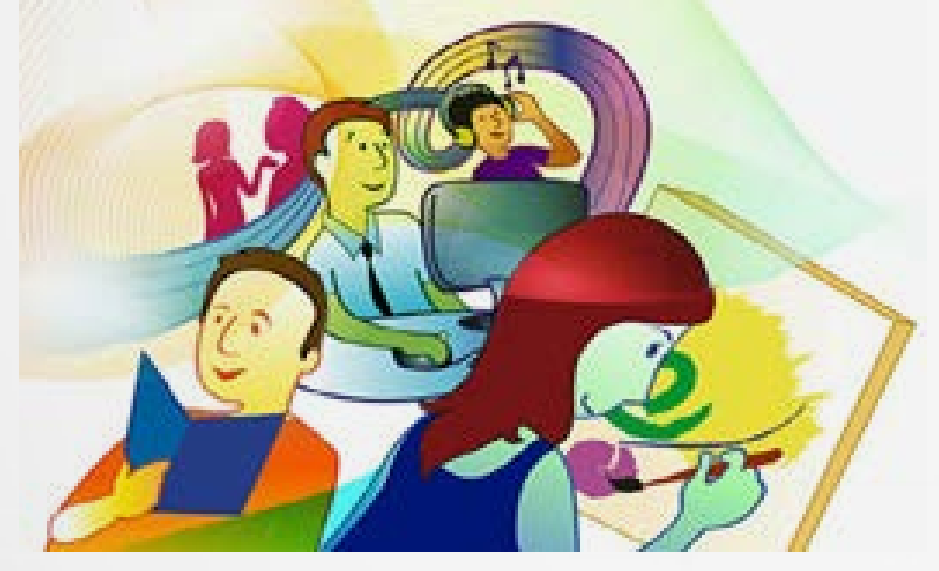

Teniendo en cuenta lo anteriormente expuesto, es posible ver que existe una preocupación por generar debates en torno a alfabetización mediática, pero también otra referida al diseño e implementación de políticas para promover procesos de alfabetización mediática. Sonia Livingstone, enumera al menos tres elementos que están al frente del debate, estos son: a) democracia, participación y ciudadanía activa; b) economía del conocimiento, competitividad y elección; y c) El aprendizaje permanente, la expresión cultural y la realización personal:

a) “La democracia, la participación y la ciudadanía activa. En una sociedad democrática, una persona alfabetizada mediáticamente es capaz de obtener una opinión informada sobre los asuntos del día y de expresar su opinión de forma individual y colectiva en los ámbitos públicos, cívicos y políticos. Una sociedad alfabetizada mediáticamente apoyaría así una esfera pública sofisticada, crítica e integradora.

b) Economía del conocimiento, la competitividad y la elección. En una economía de mercado cada vez más basada en la información, a menudo en una forma compleja y mediada, es probable que una persona alfabetizada mediáticamente tenga más que ofrecer y así pueda alcanzar un nivel superior en el lugar de trabajo, y una sociedad alfabetizada en medios sería innovadora y competitiva; lo que contribuye al mantenimiento de un amplio abanico de opciones para el consumidor.

c) El aprendizaje permanente, la expresión cultural y la realización personal. Desde nuestros entornos simbólicos fuertemente mediados, se enmarcan las opciones, valores y conocimientos que dan significado a la vida cotidiana, laalfabetización mediática contribuye a las habilidades críticas y expresivas que apoyan una vida plena y significativa, y a una sociedad informada, creativa y ética" .(Livingstone, S., et al. 2004, p.7)

Aún hoy, se debate en torno a la pertinencia del concepto de "alfabetización" respecto a otros escenarios diferentes a la adquisición de competencias de lectura y escritura. EI concepto se ha transformado y ha adquirido nuevos significados, lo que supone procesos "alfabetizadores" en diferentes dimensiones de la vida. Lo cierto es que el tipo de "alfabetización" que se mencionan en este documento (media literacy) remite al proceso de incorporación cultural de los sujetos, bien sea para hablar de competencias lecto escriturales, o metafóricamente para referir a las lecturas de contexto o de la introducción de las tecnologías de la información y la comunicación en la vida cotidiana.

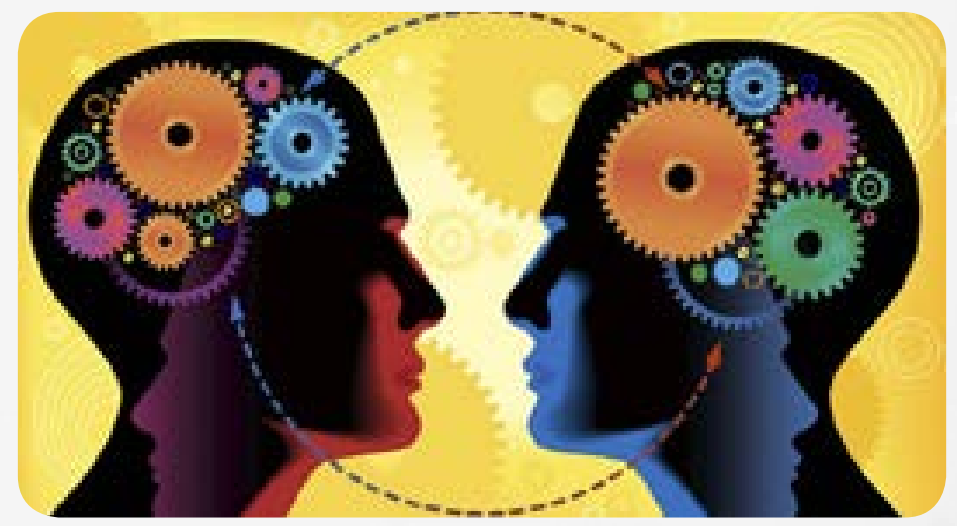

\section{Educación/comunicación}

El uso dado a la expresión educomunicación, ciertamente es diferente al empleado en los estudios sobre la materia en Iberoamérica. En la literatura anglosajona el término se relaciona más con un área denominada "instructional communication", comunicación instruccional, que persigue básicamente dos propósitos, el primero es el estudio del proceso de enseñanza de materias (cursos) específicas de la comunicación y el segundo se refiere a la enseñanza de habilidades comunicativas, tales como hablar en público. Dice Morreale (2014) 
"Los eruditos de la educomunicación, explícitamente consideran importante saber cuál es la mejor manera de enseñar a los estudiantes a mejorar sus conocimientos y habilidades de comunicación. Se centran en la enseñanza de materias de comunicación, como la interpersonal y la institucional, la comunicación en grupos pequeños, y hablar en público. Los estudiosos de la comunicación instruccional se preocupan por el papel de esta en el proceso educativo en general, por la forma de utilizar la comunicación, independientemente de la disciplina académica o el entorno de instrucción". (Sherwyn Morreale, et. Al., 2014, p.346)

Así Mismo, Sherwyn Morreale, (et.al. 2014, p 350), basados en (Cortez, Gayle, y Preiss, 2006) han identificado tres paradigmas que se han trabajado en Comunicación Educación y en Comunicación Instruccional. Estos son:

a) Paradigma proceso-producto: se basa en la noción de que los comportamientos docentes preceden, y en ellos recae la mayor responsabilidad frente al aprendizaje y rendimiento estudiantil.

b) Paradigma estudiante-mediado: se refiere a las debilidades que algunos ven en el paradigma proceso-producto, y es que el estudiante también es responsable de su propio aprendizaje.

c) El paradigma de la cultura de la escuela: se refiere principalmente a los efectos de la situación de la educación y el contexto de la enseñanza y el aprendizaje.

Los tres paradigmas enunciados se refieren a elementos distintos del fenómeno de la comunicación en los procesos de enseñanza - aprendizaje y al reconocimiento de los factores de orden endógeno y exógeno que inciden en este. El primero de ellos basado en el emisor, el segundo en el receptor y el tercero en el contexto.

Se presenta también un interés en la relación entre comunicación y pedagogía, así aparece una línea trabajo denominada CCP o "Critical Communication Pedagogy".

"Hasta ahora, la 'critical communication pedagogy' renueva nuestra atención sobre los textos y las tecnologías que utilizamos en nuestras aulas. Si bien la comunicación de muchas maneras ya aborda esta cuestión, la tradición crítica invita a hacer investigación sobre cómo nuestros recursos pedagógicos modelan y definen a los estudiantes, cómo interactuamos con los estudiantes (virtualmente y físicamente), y el tipo de información que compartimos con ellos. El paradigma crítico nos desafía a preguntarnos qué tipo de conocimiento acerca de la comunicación vale la pena conocer (y para quién y quién decide qué se conoce). (...) Así mismo, la CCP aumenta la atención hacia las cuestiones de justicia social como parte fundamental e integral de nuestro trabajo como investigadores y profesores de comunicación." (Fassett y Warren, 2010, 289)

Sprague, afirma que la comunicación, como disciplina ha relegado a la pedagogía, no ha reconocido su valor: "Ha sugerido que, en comparación con otras disciplinas afines, el campo de la comunicación parece haber marginado el trabajo pedagógico. Hay un reconocimiento actual en la educación superior que cada disciplina necesita una rama vital con una base pedagógica y curricular (...)" (Sprague, 1993, p.119). $Y$ en medio de su inconformidad sugiere una serie de pautas acerca de sus interpretaciones teóricas sobre la educación, comunicación relacionadas con la pedagogía. Dice Sprague (1993, pág., 115):

- Una disciplina específica de la pedagogía observaría que la comunicación, por definición, es un fenómeno social.

- Una disciplina específica de la pedagogía consideraría que la comunicación es un proceso continuo y complejo.

- Una disciplina específica de la pedagogía reconocería que la comunicación de voz humana se realiza, encarnada, y por lo general oralmente.

- Una disciplina específica de la pedagogía comprendería que el comportamiento asociado al discurso es inconsciente o automático. Dado que se desarrolla a través de los altavoces de la enculturación, por lo tanto no siempre se es consciente de cómo y por qué se habla.

- Una disciplina específica de la pedagogía admitiría que el discurso está ligado a la identidad cultural y personal. Nuestras teorías de comunicación reconocen ampliamente que el lenguaje refleja y afecta a la cultura. El lenguaje encarnado en el habla es un potente marcador social, así como una de las expresiones primarias de uno mismo [quizá la primera].

- Una disciplina específica de la pedagogía reconocería la relación entre comunicación y poder. Aunque el poder de la palabra siempre ha sido reconocida."

De manera complementaria a lo expuesto por Sprague, Cooks describe la relación entre la CCP y los procesos formales de instrucción, en donde cuestiona el ejercicio del poder y la autoridad.

"La CCP no está vinculada específicamente a la enseñanza en sitios formales de instrucción. Más bien, se tiene como meta una crítica de las distintas manifestaciones del conocimiento como lugares de poder y privilegio, a los usos de la comunicación para asegurar o resistir el poder y a la capacidad de las prácticas comunicativas para invitar a cambios y permitir espacios para la agencia y la intervención en las categorías de la educación y la escolarización endurecidas como prácticas formales institucionales. (...) La CCP es la pedagogía crítica en, de y para la comunicación."(Cooks, 2010, p.294-295)

Para hablar de este tema, Cooks reconoce aportes de autores de la pedagogía crítica, tales como Dewey, 1938; Livinstone, 1970; Freire, 1970; Giroux, 1988; Kozol, 1992 y McLaren, 1998, entre otros. A partir de ellos, Cooks hace tres cuestionamientos, que se resumen a continuación: 
- Algunos de los primeros escritos en la pedagogía crítica mantenían una visión bastante simplista sobre el poder contenido en el maestro y en otras figuras de autoridad y su uso de la misma en contra de los estudiantes (...) los maestros eran los opresores y los estudiantes los oprimidos. (...) (Cooks, 2010, p.297)

- Además de las batallas territoriales sobre las relaciones de poder que se determinan en las escuelas, la noción de empoderamiento de los estudiantes y maestros ha sido visto como problemático desde el punto de vista postmoderno (...) La visión del poder expresado en esta posición es la de múltiples sitios de dominación y resistencia y de posibles aberturas y espacios para la emancipación a través de la crítica de las prácticas discursivas situados tanto en el cuerpo como en la mente. (Cooks, 2010, p.298).

- La educación liberal supone que la participación es igual a educación; la pedagogía crítica asume que la participación crítica por parte de los profesores y estudiantes conduciría a la ruina de la autoridad jerárquica (y patriarcal) y a crear nuevos espacios de aprendizaje. Ninguno de los enfoques ha examinado críticamente el poder de la pedagogía en sí. "(Cooks, 2010, p.299).

\section{Reflexión final}

Seplanteandostendencias en los estudios de la relación entre la comunicación y la educación: a) comunicación en educación (comunicación instruccional) y b) la pedagogía crítica de la comunicación.

En el primero caso referida a los procesos de enseñanza aprendizaje y en el segundo al reconocimiento de los vínculos con la pedagogía que superan el escenario institucional y abarcan todos los escenarios en los que el sujeto participa de procesos educativos, pero que además tiene intereses de orden ideológico con propósitos emancipatorios, que luchan por condiciones de vida más justas.

La primera relación es de tipo instrumental pues asume a la comunicación como herramienta para facilitar los procesos de enseñanza aprendizaje y en el segundo caso la relación es de tipo ético político, en tanto los vínculos entre comunicación y pedagogía suponen que exista una preocupación por las condiciones de injusticia y pobreza en que viven los sujetos sociales.

\section{Bibliografía}

Alexander R. (2009). Towards a comparative pedagogy. University of Cambridge, Cowen, R. and Kazamias, A.M. (ed). International Handbook of Comparative Education, Springer, pp 923-942.
Buckingham, D. (2003). Media education: Literacy, learning and contemporary culture. Cambridge, MA: Polity. 232 p.

Buckingham, D. (2003). Media education: Literacy, learning and contemporary culture. Cambridge, MA: Polity.

Catt, I. E., \& Eicher-Catt, D. (Eds.). (2010). Communicology: The new science of embodied discourse. Fairleigh Dickinson Univ Press.

Catt, I.E. (2003). Gregory Bateson's 'new science' in the context of communicology. The American Journal of Semiotics, 19(1/4), 153-172.

Chen, D.-T., \& Wu, J. (2011). New media literacy in the 21th century society: Key findings, gaps and recommendations. Paper presented at the National Association for Media Literacy Education, Philadelphia, Pennsylvania, USA.

Cicero, M. S. (1876). De Oratory (J. S. Watson, Trans.). London: George Bell and Sons.

Cooks, L. (2010). The (critical) pedagogy of communication and the (critical) communication of pedagogy. The SAGE handbook of communication and instruction, 293-314.

Dale, E. (1969). Audiovisual methods in teaching.

Duncan, B. (2005). Media Literacy: Essential Survival Skills for the New Millennium. Association for Media Literacy, Ontario, Orbit, vol. 35, no. 2, P. 31-34

Fassett \&Warren (2010). In: Cooks, L. (2010). The (critical) pedagogy of communication and the (critical) communication of pedagogy. The SAGE handbook of communication and instruction, 283-292.

pedagogy. Sage Publications.

(2007). Critical communication

Giroux, H., (1992). Literacy, pedagogy, and the politics of difference. College Literature. Vol. 19 Issue 1, p1. 11p.

Hamilton D. (2009) Blurred in translation: reflections on pedagogy in public education, Pedagogy, Culture \& Society, 17:1, 5-16, DOI: 10.1080/14681360902742829 http://dx.doi. org/10.1080/14681360902742829)

Lanigan, Richard L., (1988). Phenomenology of Communication: Merleau-Ponty's Thematics in Communicology and Semiology. Pittsburgh: Duquesne University Press

, (1992). The Human Science

of Communicology. Pittsburgh, PA: Duquesne University Press

(1995). Time Binding: The Conjunction of Semiotics and Communicology. Cruzeiro Semiótico 22-25 (1995b) 325-336.

(2007). Cultura. International Journal of Philosophy of Culture and Axiology, No.8. P.p.211-216 
(2008) Communicology. In: International Encyclopedia of Communication (12 Vols.), ed. Wolfgang Donsbach. Oxford, UK and Malden, MA: Wiley-Blackwell Publishing Co.; International Communication Association, Vol. 3, p.p. 855-857.

(2010). The Verbal and Nonverbal Codes of Communicology: The Foundation of Interpersonal Agency and Efficacy: 102-128. Communicology: The New Science of Embodied Discourse, ed. Isaac E. Catt and Deborah EicherCatt. Madison, NJ: Fairleigh Dickinson University Press.

Lillywhite, H. (1952). Communication in Education. Phi Delta Kappan, p.p. 67-68

Lin, T.-B., Li, J.-Y., Deng, F., \& Lee, L. (2013). Understanding New Media Literacy: An Explorative Theoretical Framework. Educational Technology \& Society, 16 (4), 160-170.

Livingstone, S., Van Couvering, E., \& Thummin, N. (2004). Adult media literacy - A review of the research literature on behalf of Ofcom. London, UK: Office of Communications. $90 \mathrm{p}$.

Loughran, J. (2013). Pedagogy: Making Sense of the Complex Relationship Between Teaching and Learning. Ontario Institute for Studies in Education of the University of Toronto. Curriculum Inquiry 43 Published by Wiley Periodicals, Inc., 350 Main Street, Malden, MA 02148, USA, and 9600 Garsington Road, Oxford OX4 2DQ, UK, Pp. 118 - 141.

Mortimore, P. \& Watkins C., 1999, Understanding Pedagogy: And It's Impact on Learning. Ed. SAGE, 236.

Nieman (Ed), Philosophy of Education 1995 Urbana, Illinois: Philosophy of Education Society. pp. 185-194

Ohler, J., (2009). New-Media Literacies. Academe, 01902946, May/Jun, Vol. 95, Issue 3. Consulted in: http://web.b.ebscohost.com/ehost/detail/ detail? sid=8ebb8754-6eb0-448b-b8f0-fb29a69d205e $\% 40$ sessionmgr $110 \&$ vid $=0$ \&hid $=115 \&$ bdata $=\mathrm{JnNpdGU}$ 9ZWhvc3QtbGI2ZQ\%3d\%3d\#db=a2h\&AN=41232461 Consulted: 20th September, 2014

Petrilli, Susan and Augustio Ponzio (2005). Semiotics Unbounded: Interpretive Routes through the Open Network of Signs. Toronto, Buffalo, London: University of Toronto Press.

Potter, W. J. 2004. Argument for the need for a cognitive theory of media literacy. The American Behavioral Scientist; 48, 2. P.266-272

Robin, Alexander, (2001). Border Crossings: Toward a Comparative Pedagogy." Comparative Education 37, P.p. $507-523$.

Ryan, M., \& Healy, A. (2009). It's not all about school: Ways of disrupting pre-service teachers' perceptions of pedagogy and communication. Teaching and Teacher Education, 25(3), 424-429.

Salomon, G. (1981). Communication and education: Social and psychological interactions. People \& Communication.
Sandlin, J. A.,O'Malley, M. P., \& Burdick, J. 2011, Mapping the complexity of public pedagogy scholarship: 1894-2010. Review of Educational Research, \# 81, p. 173.

Sharkov, F., (2014) Communicology. In: Communicology \# 2. Ed. "Optima Project,Moscow, Russia. http://en.communicology.us/2014/01/ introductory-article-communicology-2.html)

Sherwyn Morreale, Philip Backlund \& Leyla Sparks (2014) Communication. Education and Instructional Communication: Genesis and Evolution as Fields of Inquiry, Communication Education, 63:4, 344-354. To link to this article: http://dx.doi.org/10.1080/036345 23.2014.944926

Sprague, J., (1993). Retrieving the research agenda for communication education: Askin the pedagogical questions that are 'embarrassments to theory? In: Communication, Volume 42, April, p.p. 106-122)

Tasos Kazepides (2012) Education as Dialogue, Educational Philosophy and Theory, 44:9, 913-925.

Thiessen D., Campbell, E., Gaztambide-Fernandez R., Niyozov S., Anwaruddin S., Cooke C. and Gladstone L. (2013) Perspectives on Pedagogy. Ontario Institute for Studies in Education, University of Toronto, Toronto, Ontario, Canada. P.p. 1-14.

Vavrus, F. \& Bartlett L., (2012). Comparative Pedagogies and Epistemological Diversity: Social and Materials Contexts of Teaching in Tanzania. Ed. The Comparative and International Education Society. P.634 - 658. 


\section{Notas}

[1] Inclinación innata de la materia o la mente por continuar existiendo y mejorándose. Traupman, John C. (1966). Bantam Books, ed. The New Collegiate Latin \& English Dictionary. Nueva York. ISBN 0-55325329-8. Página 52.

[2] La interdisciplinariedad no es otra cosa que la reafirmación y constante epistemológica de la reagrupación de los saberes. En la ciencia moderna, la preocupación de sus principales exponentes —Galileo, Descartes, Bacon- por la sociedad científica interdisciplinaria fue invariable. La diferencia radica sólo en que añadieron a esta agrupación interdisciplinar la necesidad de una comunicación entre las disciplinas, elemento que retoma la interdisciplinariedad a mediados del siglo XX. Fueron exponentes de estas ideas: Gottfried Wilhelm von Leibnitz y Jean Amos Komenski (Comenio). Este último propuso la pansophia, como pedagogía de la unidad, capaz de eliminar la fragmentación del saber de las disciplinas. Gusdorf G. Pasado, presente y futuro de la investigación interdisciplinaria. En: Bottomore $T$ (coord.) Interdisciplinaridad y Ciencias Humanas. Madrid: Tecnos/UNESCO. 1983; 32-52.

\section{Para citar este artículo:}

Moreno, P. Elssy (2017). Perspectivas

epistemológicas entre comunicación y educación.

Revista Luciérnaga / Comunicación , Año 9, N17. Facultad de Comunicación Audiovisual- Politécnico Colombiano Jaime Isaza Cadavid- PCJIC \& Facultad

de Ciencias de la Comunicación - Universidad

Autónoma de San Luis Potosí- UASLP. México. Págs.66-79.

DOI. 10.33571/revistaluciernaga.v9n17a6 orcid.org/0000-0001-9606-0654

OJS. http://revistas.elpoli.edu.co/index.php/luc/issue/ archive

Link. http://www.politecnicojic.edu.co/index.php/ revista-luciernaga 